\title{
Spinning higher dimensional Einstein-Yang-Mills black holes
}

\author{
Sushant G. Ghosh ${ }^{1,2, a}$, Uma Papnoi ${ }^{1, b}$ \\ ${ }^{1}$ Centre for Theoretical Physics, Jamia Millia Islamia, New Delhi 110025, India \\ ${ }^{2}$ Astrophysics and Cosmology Research Unit, School of Mathematical Sciences, University of Kwa-Zulu-Natal, \\ Private Bag 54001, Durban 4000, South Africa
}

Received: 13 March 2014 / Accepted: 30 July 2014 / Published online: 15 August 2014

(C) The Author(s) 2014. This article is published with open access at Springerlink.com

\begin{abstract}
We construct a Kerr-Newman-like spacetime starting from higher dimensional (HD) Einstein-YangMills black holes via complex transformations suggested by Newman-Janis. The new metrics are a HD generalization of Kerr-Newman spacetimes which has a geometry that is precisely that of Kerr-Newman in 4D corresponding to a YangMills (YM) gauge charge, but the sign of the charge term gets flipped in the HD spacetimes. It is interesting to note that the gravitational contribution of the YM gauge charge, in HD, is indeed opposite (attractive rather than repulsive) to that of the Maxwell charge. The effect of the YM gauge charge on the structure and location of static limit surface and apparent horizon is discussed. We find that static limit surfaces become less prolate with increase in dimensions and are also sensitive to the YM gauge charge, thereby affecting the shape of the ergosphere. We also analyze some thermodynamical properties of these BHs.
\end{abstract}

\section{Introduction}

The Kerr metric [1] is an explicit exact solution of the Einstein field equations describing a spinning black hole $(\mathrm{BH})$ in four dimensional (4D) spacetime. It is well known that a BH with non-zero spinning parameter, i.e., a Kerr BH, enjoys many interesting properties distinct from its non-spinning counterpart, i.e., from a Schwarzschild BH [2]. However, there is a surprising connection between the two BHs of Einstein theory, and this is analyzed by Newman and Janis [3]. They demonstrated that applying a complex coordinate transformation, it was possible to construct both the Kerr and KerrNewman solutions starting from the Schwarzschild metric and Reissner-Nordström metric, respectively, [3]. The KerrNewman describes the exterior of a spinning massive charged BH [4]. The Newman-Janis Algorithm (NJA) is successful

\footnotetext{
a e-mail: sghosh2@jmi.ac.in; sgghosh@gmail.com

b e-mail: uma.papnoi@gmail.com
}

in analyzing several spinning BH metrics starting from their non-spinning counterparts [5-17]. For a review on the NJA (see, e.g., [18]). However, the NJA has often be considered that there is arbitrariness and physicists considered this as an ad-hoc procedure [19]. But Schiffer et al. [20] gave a very elegant mathematical proof as to why the Kerr metric can be considered as a complex transformation of the Schwarzschild metric.

It is rather well established that higher dimensions provide a natural playground for string theory and they are also required for its consistency [21,22]. Even from the classical standpoint, it is interesting to study the higher dimensional (HD) extension of Einstein's theory, and in particular its BH solutions [23]. There seems to be a general belief that endowing general relativity with a tunable parameter, namely the spacetime dimension, should also lead to valuable insights into the nature of the theory, in particular into its most basic objects: BHs. For instance, 4D BHs are known to have a number of remarkable features, such as uniqueness, spherical topology, dynamical stability, and the laws of BH mechanics. One would like to know which of these are peculiar to $4 \mathrm{D}$, and which are true more generally. At the very least, such probings into HD will lead to a deeper understanding of classical BHs and of what space-time can do at its most extreme. There is a growing realization that the physics of HD BHs can be markedly different, and much richer than their counterparts in 4D [2,24-26], e.g., the event horizon may not be spherical in $\mathrm{HD}$ and also may have no $\mathrm{BH}$ uniqueness [22]. It is of interest to consider models based on different interacting fields including the Yang-Mills (YM). In general, it is difficult to tackle Einstein-Yang-Mills (EYM) equations because of the non-linearity both in the gauge fields and in the gravitational field. The solutions of the classical YM fields depend upon the particular ansatz one chooses. Wu and Yang [27] found static spherically symmetric solutions of the YM equations in flat space for the gauge group $\mathrm{SO}(3)$. A curved spacetime generalization of these models has been investigated by several authors (see, e.g., [28]). Indeed Yasskin [28] 
has presented an explicit procedure based on the $\mathrm{Wu}-$ Yang ansatz [27] which gives the solution of EYM rather trivially. Using this procedure, Mazharimousavi and Halilsoy [29-31] have found a sequence of static spherically symmetric HD EYM BH solutions. The remarkable feature of this ansatz is that the field has no contribution from the gradient; instead, it has a pure YM non-Abelian component. It, therefore, has only the magnetic part.

The strategy of obtaining the familiar Kerr-Newman solution, both in 4D and HD, in general relativity is based on either using the metric ansatz in the Kerr-Schild form or applying the method of complex coordinate transformation to a non-rotating charged black hole. Surprisingly, it has been demonstrated that when employing HD dimensional spacetime the two approaches lead to the same result $[32,33]$ The main purpose of this work is to apply NJA to the HD EYM BH metric previously discovered in [29-31] and the spinning HD EYM BH metric is obtained. This result shows that NJA works well also in HD spacetime. We further discuss the properties of the spinning HD EYM BH such as horizons and ergosphere. Spinning BH solutions in higher dimensions are known as Myers-Perry BHs [26]. The thermodynamical quantities associated with the spinning HD EYM BH are also calculated. Further we demonstrate that the thermodynamical quantities of this $\mathrm{BH}$ go over to the corresponding quantities of Myers-Perry BH and Kerr BH.

\section{Static BH in HD EYM theory}

We consider a $(N+1)(N+2) / 2$ parameter Lie group with structure constants $C_{(\beta)(\gamma)}^{(\alpha)}$. The gauge potentials $A_{a}^{(\alpha)}$ and the YM fields $F_{a b}^{(\alpha)}$ are related through the equation

$F_{a b}^{(\alpha)}=\partial_{a} A_{b}^{(\alpha)}-\partial_{b} A_{a}^{(\alpha)}+\frac{1}{2 \sigma} C_{(\beta)(\gamma)}^{(\alpha)} A_{a}^{(\beta)} A_{b}^{(\gamma)}$.

Then one can choose the gravity and gauge field action (EYM), which in $(N+3)$-dimensions reads $[29,30,34]$

$\mathcal{I}_{\mathcal{G}}=\frac{1}{2} \int_{M} \mathrm{~d} x^{N+3} \sqrt{-g}\left[R-\sum_{\alpha=1}^{(N+1)(N+2) / 2} F_{a b}^{(\alpha)} F^{(\alpha) a b}\right]$.

Here, $g=\operatorname{det}\left(g_{a b}\right)$ is the determinant of the metric tensor, $R$ is the Ricci Scalar and $A_{a}^{(\alpha)}$ are the gauge potentials. We note that the internal indices $\{\alpha, \beta, \gamma, \ldots\}$ do not differ whether in covariant or in contravariant form. We introduce the $\mathrm{Wu}-$ Yang ansatz in $(N+3)$ dimensions [29-31] as

$$
\begin{aligned}
& A^{(\alpha)}=\frac{Q}{r^{2}}\left(x_{i} \mathrm{~d} x_{j}-x_{j} \mathrm{~d} x_{i}\right) \\
& 2 \leq i \leq N+2, \\
& 1 \leq j \leq i-1, \\
& 1 \leq(\alpha) \leq(N+1)(N+2) / 2,
\end{aligned}
$$

where the super index $\alpha$ is chosen according to the values of $i$ and $j$ in order and we choose $\sigma=Q$ [29-31]. The Wu-Yang solution appears to be highly non-linear because of mixing between spacetime indices and gauge group indices. However, it is linear as expressed in the non-linear gauge fields because a purely magnetic gauge charge is chosen along with a position-dependent gauge field transformation [28]. The YM field 2 form is defined by the expression

$F^{(\alpha)}=\mathrm{d} A^{(\alpha)}+\frac{1}{2 Q} C_{(\beta)(\gamma)}^{(\alpha)} A^{(\beta)} \wedge A^{(\gamma)}$.

The integrability conditions

$\mathrm{d} F^{(\alpha)}+\frac{1}{Q} C_{(\beta)(c)}^{(\alpha)} A^{(\beta)} \wedge F^{(\gamma)}=0$,

as well as the YM equations

$\mathrm{d} * F^{(\alpha)}+\frac{1}{Q} C_{(\beta)(\gamma)}^{(\alpha)} A^{(\beta)} \wedge * F^{(\gamma)}=0$,

are all satisfied. Here $d$ is the exterior derivative, $\wedge$ stands for the wedge product and $*$ represents Hodge duality. All these are in the usual exterior differential forms notation.

Variation of the action with respect to the space-time metric $g_{a b}$ yields the EYM equations

$G_{a b}=T_{a b}$,

where the gauge stress-energy tensor is

$T_{a b}=\sum_{\alpha=1}^{(N+1)(N+2) / 2}\left[2 F_{a}^{(\alpha) \lambda} F_{b \lambda}^{(\alpha)}-\frac{1}{2} F_{\lambda \sigma}^{(\alpha)} F^{(\alpha) \lambda \sigma} g_{a b}\right]$.

In general, it is difficult to solve the EYM (7). However, the Wu-Yang ansatz [27] facilitates obtaining the solution.

The metric for the HD EYM BH [31] obtained using the Wu-Yang ansatz [27] is given by

$\mathrm{d} s^{2}=f(r) \mathrm{d} t^{2}-f(r)^{-1} \mathrm{~d} r^{2}-r^{2} \mathrm{~d} \Omega_{N+1}^{2}$,

with

$f(r)=1-\frac{\mu}{r^{N}}-\frac{N}{(N-2)} \frac{Q^{2}}{r^{2}}, \quad N \neq 2$,

where

$$
\begin{aligned}
\mathrm{d} \Omega_{N+1}^{2}= & \mathrm{d} \theta_{1}^{2}+\sin ^{2} \theta_{1} \mathrm{~d} \theta_{2}^{2}+\sin ^{2} \theta_{1} \sin ^{2} \theta_{2} \mathrm{~d} \theta_{3}^{2}+\cdots \\
& +\left[\left(\prod_{j=1}^{N} \sin ^{2} \theta_{j}\right) \mathrm{d} \theta_{N+1}^{2}\right],
\end{aligned}
$$

where $\mu$ is the integration constant, which can be related to mass $M$ and $D=N+3$ is the number of spacetime 
dimensions. Since $T_{a b}$ go as $r^{-4}$ (the same as for Maxwell field in 4D), interestingly for all $D \geq 6$. That is why its contribution in $f(r)$ is the same for all $D \geq 6$ as in ReissnerNordström. There is, however, an important difference in the sign before $Q^{2} / r^{2}$ term. In the 4D case, it is exactly like a Reissner-Nordström BH, i.e., positive. In contrast to $4 \mathrm{D}$, the sign before $Q^{2} / r^{2}$ is negative for $D \geq 6$. On the other hand, if the YM gauge charge is switched off ( $Q=0)$, the metric (9) reduces to the well-known Schwarzschild-Tanghelini metric [2]. In addition if $N=1$, one may note that it reduces to the Schwarzschild metric. When $N=1$, the metric (9) is exactly that of a Reissner-Nordström BH with $Q$ as the YM gauge charge.

\section{Spinning HD EYM BH via NJA}

We want to derive the axially symmetric spinning analog of the static spherically symmetric EYM BH adapting Newman-Janis [3] complex transformation. Newman et al. [3] discovered curious derivations of stationary, spinning metric solutions from static, spherically symmetric solutions in 4D Einstein theory. In order to derive spinning HD EYM $\mathrm{BH}$, we start with the non-spinning version of the HD EYM BH metric (9), with $f(r)$ given by

$f(r)=1-\frac{\mu}{r^{N}}-\frac{Q_{0}^{2}}{r^{2}}, \quad Q_{0}^{2}=\frac{N}{(N-2)} Q^{2}$,

as a seed solution to construct its spinning counterpart. Following Newman and Janis [3] the first step is to write the metric (9) in advanced Eddington-Finkelstein coordinates by the following coordinate transformation:

$\mathrm{d} u=\mathrm{d} t-f(r)^{-1} \mathrm{~d} r$,

we obtain

$\mathrm{d} s^{2}=f(r) \mathrm{d} u^{2}+2 \mathrm{~d} u \mathrm{~d} r-r^{2} \mathrm{~d} \Omega_{N+1}^{2}$.

The metric (11) can be written in terms of a null veiltrad $Z^{a}=$ $\left(l^{a}, n^{a}, m_{1}^{a}, \bar{m}_{1}^{a}, m_{2}^{a}, \bar{m}_{2}^{a}, \ldots, m_{(N+1) / 2}^{a}, \bar{m}_{(N+1) / 2}^{b}\right)$, $[3,14]$ as

$$
\begin{aligned}
g^{a b}= & l^{a} n^{b}+l^{b} n^{a}-m_{1}^{a} \bar{m}_{1}^{b}-m_{2}^{a} \bar{m}_{2}^{b}, \ldots, \\
& -m_{(N+1) / 2}^{a} \bar{m}_{(N+1) / 2}^{b},
\end{aligned}
$$

where the null vieltrad are

$$
\begin{aligned}
l^{a}= & \delta_{r}^{a}, \\
n^{a}= & {\left[\delta_{u}^{a}-\frac{1}{2}\left(1-\frac{\mu}{r^{N}}-\frac{Q_{0}^{2}}{r^{2}}\right) \delta_{r}^{a}\right], } \\
m_{k}^{a}= & \frac{1}{\sqrt{2} r \sin \theta_{1} \sin \theta_{2}, \ldots, \sin \theta_{(k-1)}} \\
& \times\left(\delta_{\theta_{k}}^{a}+\frac{i}{\sin \theta_{k}} \delta_{\theta_{(k+1)}}^{a}\right),
\end{aligned}
$$

where $D=N+3$, is spacetime dimension and $k$ is used to denote the number of vectors which take values $1,2,3, \ldots,(N+1) / 2$, e.g., in $6 \mathrm{D} k$ is 2 , which corresponds to the following vector:

$$
\begin{aligned}
& m_{1}^{a}=\frac{1}{\sqrt{2} r}\left(\delta_{\theta_{1}}^{a}+\frac{i}{\sin \theta_{1}} \delta_{\theta_{2}}^{a}\right), \\
& m_{2}^{a}=\frac{1}{\sqrt{2} r \sin \theta_{1}}\left(\delta_{\theta_{2}}^{a}+\frac{i}{\sin \theta_{2}} \delta_{\theta_{3}}^{a}\right) .
\end{aligned}
$$

Here we assume $N$ is odd, i.e., the spacetime dimension $D$ is even. However, the final result is independent of this assumption. Note that $l^{a}$ and $n^{a}$ are real, $m_{k}^{a}, \bar{m}_{k}{ }^{a}$ are mutually complex conjugate. This vieltrad is orthonormal and obeys metric conditions. We have

$$
\begin{aligned}
& l_{a} l^{a}=n_{a} n^{a}=\left(m_{k}\right)_{a}\left(m_{k}\right)^{a}=\left(\bar{m}_{k}\right)_{a}\left(\bar{m}_{k}\right)^{a}=0, \\
& l_{a}\left(m_{i}\right)^{a}=l_{a}\left(\bar{m}_{k}\right)^{a}=n_{a}\left(m_{k}\right)^{a}=n_{a}\left(\bar{m}_{k}\right)^{a}=0, \\
& l_{a} n^{a}=1, \quad\left(m_{k}\right)_{a}\left(\bar{m}_{k}\right)^{a}=1 .
\end{aligned}
$$

Now we allow for some $r$ factor in the null vectors to take on complex values. Following [5,11], we rewrite the null vectors in the form

$l^{a}=\delta_{r}^{a}$,

$n^{a}=\left[\delta_{u}^{a}-\frac{1}{2}\left(1-\frac{\mu}{2 r^{N-1}}\left[\frac{1}{r}+\frac{1}{\bar{r}}\right]-\frac{Q_{0}^{2}}{r \bar{r}}\right) \delta_{r}^{a}\right]$,

$m_{k}^{a}=\frac{1}{\sqrt{2} \bar{r} \sin \theta_{1} \sin \theta_{2} \ldots \sin \theta_{(k-1)}}\left(\delta_{\theta_{k}}^{a}+\frac{i}{\sin \theta_{k}} \delta_{\theta_{(k+1)}}^{a}\right)$,

with $\bar{r}$ being the complex conjugate of $r$. In 4D there is only one possible spinning axisymmetric spacetime, and there is therefore only one angular momentum parameter. In HD there are several choices of the spinning axis and there is a multitude of angular momentum parameters, each referring to a particular spinning plane. We concentrate on the simplest case for which there is only one angular momentum parameter, which we shall denote by $a$. Next we perform a similar complex coordinate transformation, in the HD, as used by Newman and Janis [3] by defining a new set of coordinates $\left(u^{\prime}, r^{\prime}, \theta_{i}^{\prime}\right)$, where $i=1, \ldots,(N+1) / 2$ by the relations

$$
\begin{array}{r}
x^{\prime a}=x^{a}+i a\left(\delta_{r}^{a}-\delta_{u}^{a}\right) \cos \theta_{1} \\
\rightarrow\left\{\begin{array}{l}
u^{\prime}=u-i a \cos \theta_{1}, \\
r^{\prime}=r+i a \cos \theta_{1}, \\
\theta_{i}^{\prime}=\theta_{i}
\end{array}\right.
\end{array}
$$

Simultaneously let the null vieltrad vectors $Z^{a}$ undergo a transformation $Z^{a}=Z^{\prime a} \partial x^{\prime a} / \partial x^{b}$ in the usual way; we obtain 


$$
\begin{aligned}
l^{a}= & \delta_{r}^{a}, \\
n^{a}= & {\left[\delta_{u}^{a}-\frac{1}{2}\left(1-\frac{\mu}{r^{N-2} \Sigma}-\frac{Q_{0}^{2}}{\Sigma}\right) \delta_{r}^{a}\right], } \\
m_{k}^{a}= & \frac{1}{\sqrt{2}\left(r+i a \cos \theta_{1}\right) \sin \theta_{1} \sin \theta_{2}, \ldots, \sin \theta_{(k-1)}} \\
& \times\left(i a\left(\delta_{u}^{a}-\delta_{r}^{a}\right) \sin \theta_{1}+\delta_{\theta_{k}}^{a}+\frac{i}{\sin \theta_{k}} \delta_{\theta_{(k+1)}}^{a}\right),
\end{aligned}
$$

where we have dropped the primes. From the new null vieltrad, a new metric is discovered using (12), which can be written as

$$
\begin{aligned}
\mathrm{d} s^{2}= & \left(1-\frac{\mu}{r^{N-2} \Sigma}-\frac{Q_{0}^{2}}{\Sigma}\right) \mathrm{d} u^{2}+2 \mathrm{~d} u \mathrm{~d} r-2 a \sin ^{2} \theta_{1} \mathrm{~d} r \mathrm{~d} \theta_{2} \\
& -\Sigma \mathrm{d} \theta_{1}^{2}-\left[\left(r^{2}+a^{2}\right)+\left(\frac{\mu}{r^{N-2} \Sigma}+\frac{Q_{0}^{2}}{\Sigma}\right) a^{2} \sin ^{2} \theta_{1}\right] \\
& \times \sin ^{2} \theta_{1} \mathrm{~d} \theta_{2}^{2}-2 a\left(\frac{\mu}{r^{N-2} \Sigma}+\frac{Q_{0}^{2}}{\Sigma}\right) \sin \theta_{1}^{2} \mathrm{~d} u \mathrm{~d} \theta_{2} \\
& -r^{2} \mathrm{~d} \Omega_{N-1}^{2},
\end{aligned}
$$

where $\Sigma=r^{2}+a^{2} \cos \theta_{1}^{2}$. Thus, we have obtained a spinning $\mathrm{BH}$ corresponding to HD EYM BH. Also note that the derived HD metric (14) via NJA is in Kerr-like coordinates [6]. A further simplification is made on taking a coordinate transformation as in Ref. [6]. This transformation leaves only one off-diagonal element and we arrive at the following:

$$
\begin{aligned}
\mathrm{d} s^{2}= & \left(\frac{\Delta-a^{2} \sin ^{2} \theta_{1}}{\Sigma}\right) \mathrm{d} t^{2}-\frac{\Sigma}{\Delta} \mathrm{d} r^{2}+2 a \\
& \times\left[1-\left(\frac{\Delta-a^{2} \sin ^{2} \theta_{1}}{\Sigma}\right)\right] \mathrm{d} t \mathrm{~d} \theta_{2}-\Sigma \mathrm{d} \theta_{1}^{2} \\
& -\left[\Sigma+a^{2} \sin ^{2} \theta_{1}\left(2-\frac{\Delta-a^{2} \sin ^{2} \theta_{1}}{\Sigma}\right)\right] \\
& \times \sin ^{2} \theta_{1} \mathrm{~d} \theta_{2}^{2}-r^{2} \cos ^{2} \theta_{1} \mathrm{~d} \Omega_{N-1}^{2},
\end{aligned}
$$

where on substituting back the value of $Q_{0}$ in terms of $Q, \Delta$ reads

$\Delta=r^{2}+a^{2}-\frac{\mu}{r^{N-2}}-\frac{N}{(N-2)} Q^{2}$.

Equation (15) is in Boyer-Lindquist coordinates. Here, we have also introduced

$\Delta=a^{2} \sin ^{2} \theta_{1}+\Sigma G\left(r, \theta_{1}\right)$,

$G\left(r, \theta_{1}\right)=\frac{\Delta-a^{2} \sin ^{2} \theta_{1}}{\Sigma}$.

Thus, we are able to generate a HD axisymmetric solution starting with HD static spherically symmetric EYM BH solutions using the approach originally proposed by NewmanJanis [3], i.e., we have an explicit YM gauge charged HD spinning BH solution. One can see that HD metric (15) shows the behavior of a metric produced by a spinning charged source. In the limit $N=1$, the geometry of solution (15) is precisely of the Kerr-Newman form [6] and the charge that determines the geometry is the YM gauge charge. Thus we have an exact HD Kerr-Newman-like solution, but $Q$ corresponds to the magnetic charge. Hereafter, we refer to the solutions (15) as spinning HD EYM BH solutions. For a vanishing YM gauge charge $Q=0$, one recovers Myers-Perry $\mathrm{BH}$ solution discussed in [26]. The Reissner-Nordström BH are recovered in the limit $a=0$ and $N=1$. The HD EYM $\mathrm{BH}$ [31] are discovered for the vanishing spinning parameter $a=0$. It is nice to see that the HD metric (15) gives an allcorrect limit. We still have to ensure that the HD metric (15) indeed solves the EYM equations (7). The NJA is widely used in general relativity and is correct for 4D. If $D \neq 4$, the trace of the EMT (8) tensor is not equal to zero, then $R \neq 0$. This makes the problem of rotation in the HD-EYM case more complicated. It is well known that there is also a dispute over the existence of Kerr-Newman solutions in higher dimensions [32]. The Kerr-Newman solution in general relativity is obtained either using the metric ansatz in the Kerr-Schild form or applying the method of a complexcoordinate transformation to a non-rotating charged black hole. However, it turns out that the two procedures lead to the same metric [33]. We have presented a HD version of a rotating EYM BH solution using the complex transformation and we demonstrated that it has all properties of a rotating $\mathrm{BH}$. We have justified that the properties of metric (15) are very similar to Kerr/Kerr-Newman. In particular, we have also calculated an event horizon and time-like limit surface and they are also similar to a Myers-Perry BH.

Equation (15) has parameters $\mu$ and $a$, which are, respectively, related to the mass $(M)$ and the angular momentum $(J)$ via the relations

$M=\frac{(N+1)}{16 \pi} A_{N+1} \mu, \quad J=\frac{1}{8 \pi} A_{N+1} \mu a$

and

$\frac{M}{J}=\frac{(N+1)}{2} a$

The determinant $g$ of the metric (15) gives

$\sqrt{-g}=\sqrt{\gamma} \Sigma r^{N-1} \sin \theta \cos ^{N-1} \theta$

and $A_{N+1}$ is the area of a unit $(N+1)$ sphere, which is given by

$$
\begin{aligned}
A_{N+1}= & \int_{0}^{2 \pi} \mathrm{d} \theta_{2} \int_{0}^{\pi} \sin \theta_{1} \cos ^{N-1} \theta_{1} \mathrm{~d} \theta_{1} \\
& \times \prod_{i=3}^{N-1} \int_{0}^{\pi} \sin ^{(N-1)-i} \theta_{i} \mathrm{~d} \theta_{i}=\frac{2 \pi^{(N+2) / 2}}{\Gamma(N+2) / 2} .
\end{aligned}
$$



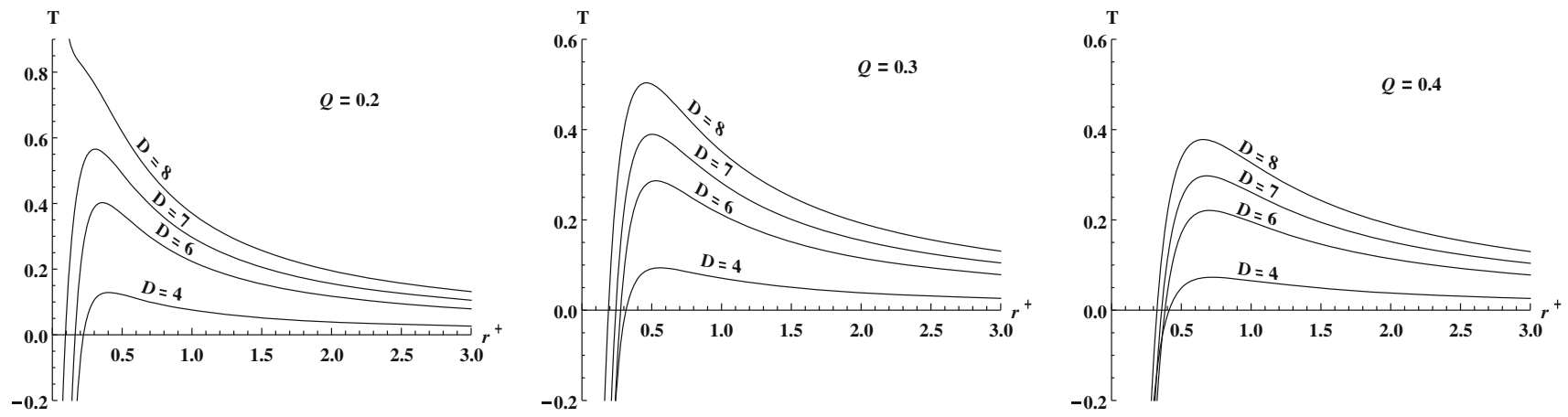

Fig. 1 The temperature profile shown as function of $r^{+}$for different dimensions $D$ with three different values of the YM gauge charge parameter $Q$
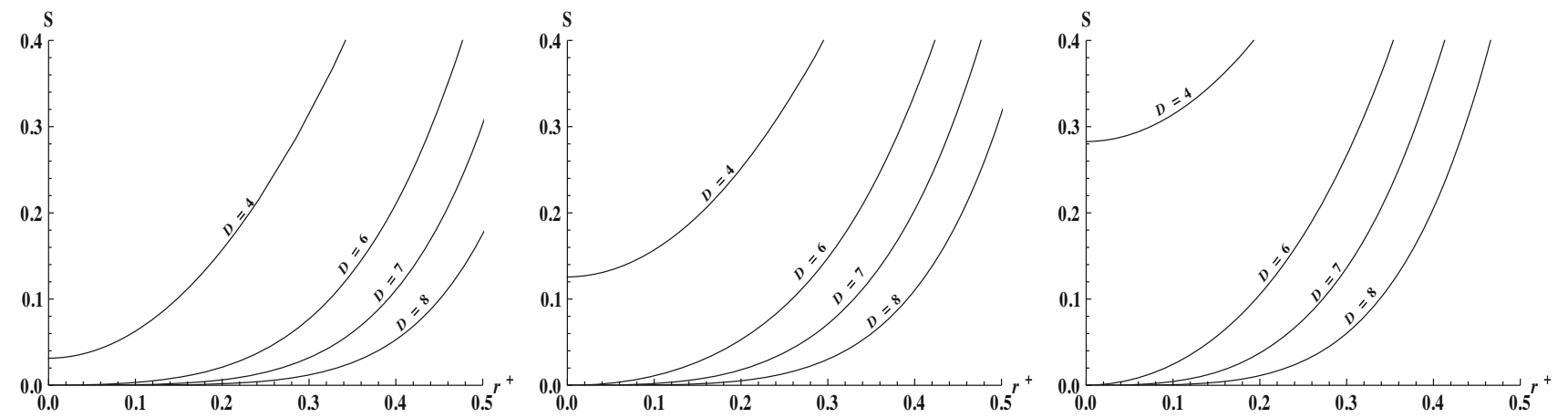

Fig. 2 The behavior of entropy as a function of horizon radius $r^{+}$for different dimensions $D$ with three different values of rotation parameter $a$

The angular velocity at the horizon is given by

$$
\Omega_{\mathrm{H}}=\frac{a}{r^{+2}+a^{2}} .
$$

The area of the event horizon (EH) for the metric (15) can be given by the standard definition of the horizon area [35] as:

$$
A_{\mathrm{H}}=\int_{\rho\left(r=r^{+}\right)} \sqrt{\eta} \mathrm{d} \theta_{1} \mathrm{~d} \theta_{2} d^{N-1} \rho,
$$

which trivially solves to

$$
A_{H}=r^{+N-1}\left(r^{+2}+a^{2}\right) A_{N+1} \text {. }
$$

The entropy of the BH typically satisfies the area law of the entropy, which states that the entropy of the $\mathrm{BH}$ is one fourth of the area of EH [36]. The horizon area and the surface gravity of the solution are related to the entropy and the temperature, respectively, $S=A_{H} / 4$ and $T=\kappa / 2 \pi$. Thus the expression for the entropy and the temperature of the spinning HD EYM BH on the horizon are

$$
\begin{aligned}
& S=\frac{r^{+N-1}\left(r^{+2}+a^{2}\right) A_{N+1}}{4}, \\
& T=\frac{(N-2)\left(r^{+2}+a^{2}\right)+2 r^{+2}-N Q^{2}}{4 \pi r^{+}\left(r^{+2}+a^{2}\right)} .
\end{aligned}
$$

In the appropriate limit the physical quantities derived above reproduce the corresponding qualities of a Myers-Perry BH when $Q=0$, of a Kerr-Newman BH if $N=1$ and when both $Q=0$ and $N=1$, we get these quantities associated with Kerr BH. In Figs. 1 and 2, we plot the temperature and the entropy of the spinning HD EYM BH, respectively. It is interesting to note from Fig. 1 that at a low value of the YM gauge charge the temperature is at maximum in HD but as $Q$ is increased the temperature starts to increase from a minimum value and on reaching a maximum value, it starts to decay. We note that the rate of decrease in temperature slows down with the increase in $Q$. The entropy for the 4D case, $N=1$, is always positive even for vanishing horizon radius, $r^{+}=0$, but it is zero in the HD case for $r^{+}=0$. The dependence of the entropy on the horizon radius, $r^{+}$, is shown in Fig. 2, which also confirms the area law for our solution, i.e., the entropy is increasing with the radius of the horizon.

\section{Horizon properties}

It is natural to discuss not only spinning BH solutions but their various properties. It is known that the structure of a spinning BH is very different from that of a stationary $\mathrm{BH}$. The $\mathrm{EH}$ of a spinning $\mathrm{BH}$ is smaller than the $\mathrm{EH}$ of an other- 
wise identical but non-spinning one. Similar to Kerr solutions in asymptotically flat spacetimes the above metric has two types of horizon-like hypersurface: a stationary limit surface (SLS) and an EH. Within the stationary limit, no particles can remain at rest, even though they are outside the EH. We shall explore the two horizons SLS and EH of spinning HD EYM BH, and also we shall discuss the effects which come from the YM gauge charge and also the effects due to the spacetime dimension.

\subsection{SLS and EH}

Let us now address the horizon properties of the solution, beginning with SLS. The SLS is the boundary of the region in which an observer traveling along a time-like curve can follow the orbits of the asymptotic time translation Killing vector $\partial / \partial t$ and so remain stationary with respect to infinity. Physical observers cannot follow the orbit of $\partial / \partial t$ beyond the EH surface since in that region they are space-like orbits. On this surface the Killing vector $\partial / \partial t$ is null. They are surfaces of infinite redshift, and for the spinning HD EYM BH one requires that the prefactor $g_{t t}$ of the $d t^{2}$ term in the metric vanish. It follows that the SLS will satisfy

$r^{N}+\left[a^{2} \cos ^{2} \theta-\frac{N}{(N-2)} Q^{2}\right] r^{N-2}-\mu=0$.

On the other hand, surfaces at which a particle traveling on a time-like curve from a point on or inside the surface cannot get outside the surface and so cannot get out to infinity is an EH. An EH is a solution of $\Delta=0$ and thus it must satisfy

$r^{N}+\left[a^{2}-\frac{N}{(N-2)} Q^{2}\right] r^{N-2}-\mu=0$.

\subsubsection{D case}

When $N=1$, i.e., in $4 \mathrm{D}$, recalling that $\mu=2 M$, we recover the well-known results for the Kerr-Newman metric:

$$
\begin{aligned}
\mathrm{d} s^{2}= & \left(1-\frac{2 M r}{\Sigma}+\frac{Q^{2}}{\Sigma}\right) \mathrm{d} t^{2}-\frac{\Sigma}{\Delta} \mathrm{d} r^{2}-\Sigma \mathrm{d} \theta^{2} \\
& -\left[\left(r^{2}+a^{2}\right)+\left(\frac{2 M r}{\Sigma}-\frac{Q^{2}}{\Sigma}\right) a^{2} \sin ^{2} \theta\right] \sin ^{2} \theta \mathrm{d} \phi^{2} \\
& +2 a\left(\frac{2 M r}{\Sigma}-\frac{Q^{2}}{\Sigma}\right) \sin ^{2} \theta \mathrm{d} t \mathrm{~d} \phi,
\end{aligned}
$$

with $\theta_{1}=\theta, \theta_{2}=\phi$ and in (15) $\mu=2 M$. Equation (22), for $N=1$, is simplified to

$\Delta=r^{2}+a^{2}-2 M r+Q^{2}$,

which admits a solution $r^{ \pm}$, identified as outer and inner EH. The EH of (23) are $r^{ \pm} \mathrm{EH}=M \pm \sqrt{\left(M^{2}-Q^{2}\right)-a^{2}}$.

If $a^{2}<\left(M^{2}-Q^{2}\right)$, there exist two horizons, when $a^{2} \rightarrow$ $\left(M^{2}-Q^{2}\right)$, the two horizons coincide, i.e., the extremal case and if $a^{2}>\left(M^{2}-Q^{2}\right)$, then there exists no horizon, i.e., one has a naked singularity. For SLS, $N=1$ in (21), it reduces to

$r^{2}-2 M r+a^{2} \cos ^{2} \theta+Q^{2}=0$,

which trivially solves to

$r^{ \pm} \mathrm{SLS}=M \pm \sqrt{\left(M^{2}-Q^{2}\right)-a^{2} \cos ^{2} \theta}$.

These are regular outer and inner SLSs for a Kerr-Newman BH when $a^{2} \cos ^{2} \theta<\left(M^{2}-Q^{2}\right)$, and further in the nonspinning limit $a \rightarrow 0$, SLS and EH coincide with

$r^{ \pm}=M \pm \sqrt{M^{2}-Q^{2}}$,

which are outer and inner EH of Reissner-Nordström BH. Thus the Kerr-Newman BH, in the limit $a \rightarrow 0$, degenerates to a Reissner-Nordström BH.

\subsection{2 $6 D$ case}

Equation (22) for the $N=3$ or $6 D$ case reduces to

$r^{3}+\left(a^{2}-3 Q^{2}\right) r-\mu=0$,

which gives $\mathrm{EH}$ as

$$
\begin{aligned}
r_{E H}^{+}= & \frac{\left(27 \mu+\sqrt{729 \mu^{2}+\delta}\right)^{1 / 3}}{3 \times 2^{1 / 3}} \\
& -\frac{2^{1 / 3}\left(a^{2}-3 Q^{2}\right)}{\left(27 \mu+\sqrt{729 \mu^{2}+\delta}\right)^{1 / 3}},
\end{aligned}
$$

with $\delta=4\left(3 a^{2}-9 Q^{2}\right)^{3}$.

Equation (21) reduces to

$r^{3}+\left(a^{2} \cos ^{2} \theta-3 Q^{2}\right) r-\mu=0$,

which can be solved to

$$
\begin{aligned}
r_{S L S}^{+}= & \frac{\left(27 \mu+\sqrt{729 \mu^{2}+\delta}\right)^{1 / 3}}{3 \times 2^{1 / 3}} \\
& -\frac{2^{1 / 3}\left(a^{2} \cos ^{2} \theta-3 Q^{2}\right)}{\left(27 \mu+\sqrt{729 \mu^{2}+\delta}\right)^{1 / 3}},
\end{aligned}
$$

with $\delta=4\left(3 a^{2} \cos ^{2} \theta-9 Q^{2}\right)$.

\subsubsection{D Case}

Equation (22), for 7D, reduces to

$$
r^{4}+\left(a^{2}-2 Q^{2}\right) r^{2}-\mu=0 .
$$



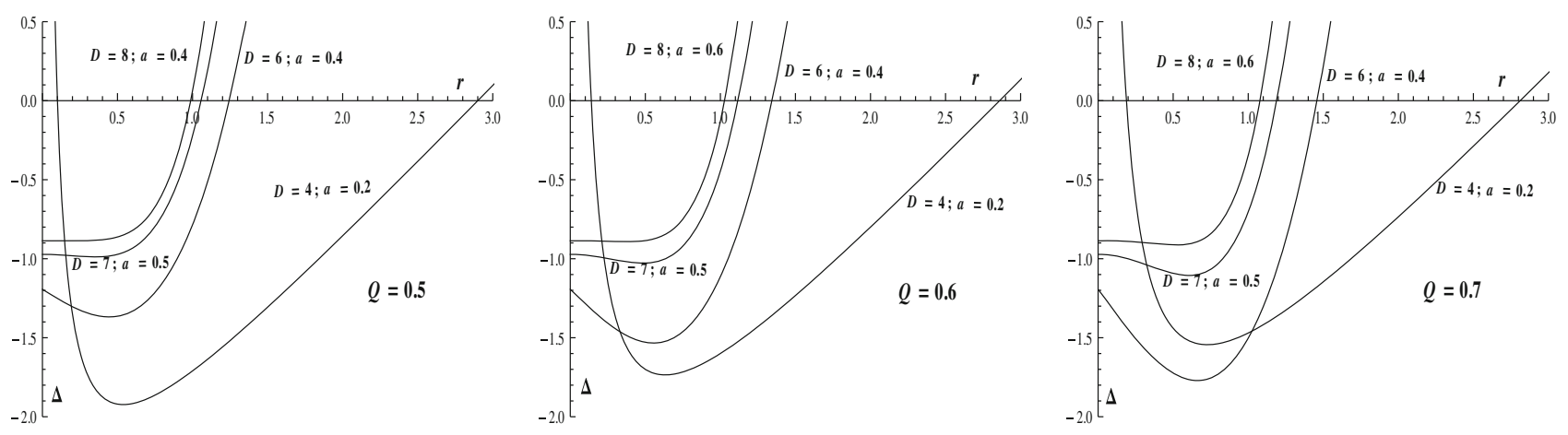

Fig. 3 Plot of $\Delta(r)$ to show the behavior of horizon of spinning HD EYM BH for different dimensions $D$ with three different values of YM gauge charge parameter $Q$. Here we choose $M=1$

So, we get the EH as

$r_{E H}^{+}=\sqrt{\frac{1}{2} \sqrt{4 \mu+\delta_{1}}+\frac{1}{2}\left(2 Q^{2}-a^{2}\right)}$,

with $\delta_{1}=a^{4}+4 Q^{2}-4 a^{2} Q^{2}$.

Equation (21) can be written as

$r^{4}+\left(a^{2} \cos ^{2} \theta-2 Q^{2}\right) r^{2}-\mu=0$,

which admits the solution

$r^{+}$SLS $=\sqrt{\frac{1}{2} \sqrt{4 \mu+\delta_{2}}+\frac{1}{2}\left(2 Q^{2}-a^{2} \cos ^{2} \theta\right)}$,

with $\delta_{2}=a^{4} \cos ^{4} \theta-4 a^{2} \cos ^{2} \theta+4 Q^{2}$.

Thus, the SLS and EH depends on the spacetime dimension. For HD we note that

$\lim _{r \rightarrow 0} \Delta(r)=-\mu<0, \quad \lim _{r \rightarrow+\infty} \Delta(r)=\infty, \quad \Delta^{\prime}(r) \geq 0$.

It is seen that $\Delta^{\prime}(r) \geq 0$ for $D \geq 6$. However, it is seen that Eqs. (21) and (22) have just one positive root for HD $(D \geq 6)$, i.e., just one EH and SLS in HD. This means that there is no extremal spinning $\mathrm{BH}$ when $D \geq 6$. The effect of the YM gauge charge on the horizon is shown in Fig. 3. It turns out that the radius of EH is decreasing with the spacetime dimension. On the other hand, it increases with the value of the YM gauge charge. One gets the same result for SLS and we do not present them here.

\subsection{Ergosphere}

For the Schwarzschild and Reissner-Nordström BH, it is possible that a traveler can approach arbitrarily close to the $\mathrm{EH}$ while remaining stationary with respect to infinity. This is not the case for the Kerr/Kerr-Newman BH. The spinning $\mathrm{BH}$ drags the surrounding region of spacetime, causing the traveler to spin regardless of any arbitrarily large thrust that he can provide. The ergoregion is the region in which this happens and is bounded by the ergosphere. On plotting the SLS and EH for a spinning HD EYM BH, it can be verified that the SLS always lies outside the EH for all dimensions $D$. The ergosphere is plotted in Figs. 4 and 5. The ergosphere is defined to be the place where the vectors $\partial t$ parallel to the time-axis are not time-like but space-like. An ergosphere, thus, is a region of spacetime where no observer can remain still with respect to the coordinate system in question. Thus the ergosphere of a spinning HD EYM BH, as in Kerr/KerrNewman BH, is bounded by the EH on the inside and an oblate spheroid SLS, which coincides with the EH at the poles and is noticeably wider around the equator. It is the region of spacetime where time-like geodesics remain stationary and time-like particles can have negative energy relative to infinity. It is theoretically possible to extract energy and matter from the BH from the ergosphere [37]. In Fig. 4 we show the dependence of the shape of the ergosphere on the YM gauge charge. It is noticed that for $D=4$ the shape of the ergosphere is increasing with the increase in the YM gauge charge while decreasing for $D \geq 6$. This shows that the shape of the ergosphere is sensitive to the YM gauge charge. In Fig. 5 the variation of the shape of ergosphere with $a$ is shown. We note that the relative shape of the ergosphere becomes more prolate, thereby increasing the area of the ergosphere with rotation parameter $a$, i.e., the faster the $\mathrm{BH}$ rotates, the more the ergosphere grows. This may have direct consequences on the Penrose energy extraction process [37].

\section{Conclusion}

In this paper, we have extended NJA in order to construct a spinning BH from HD EYM BH. The method does not use a field equation but works on the HD spherical solution to generate spinning solutions. The algorithm is very useful since it directly allows us to construct a spinning $\mathrm{BH}$ in HD, which otherwise could be extremely cumbersome. Originally, the NJA was applied to the Reissner-Nordström solution to obtain a Kerr-Newman solution [5]. The metric 

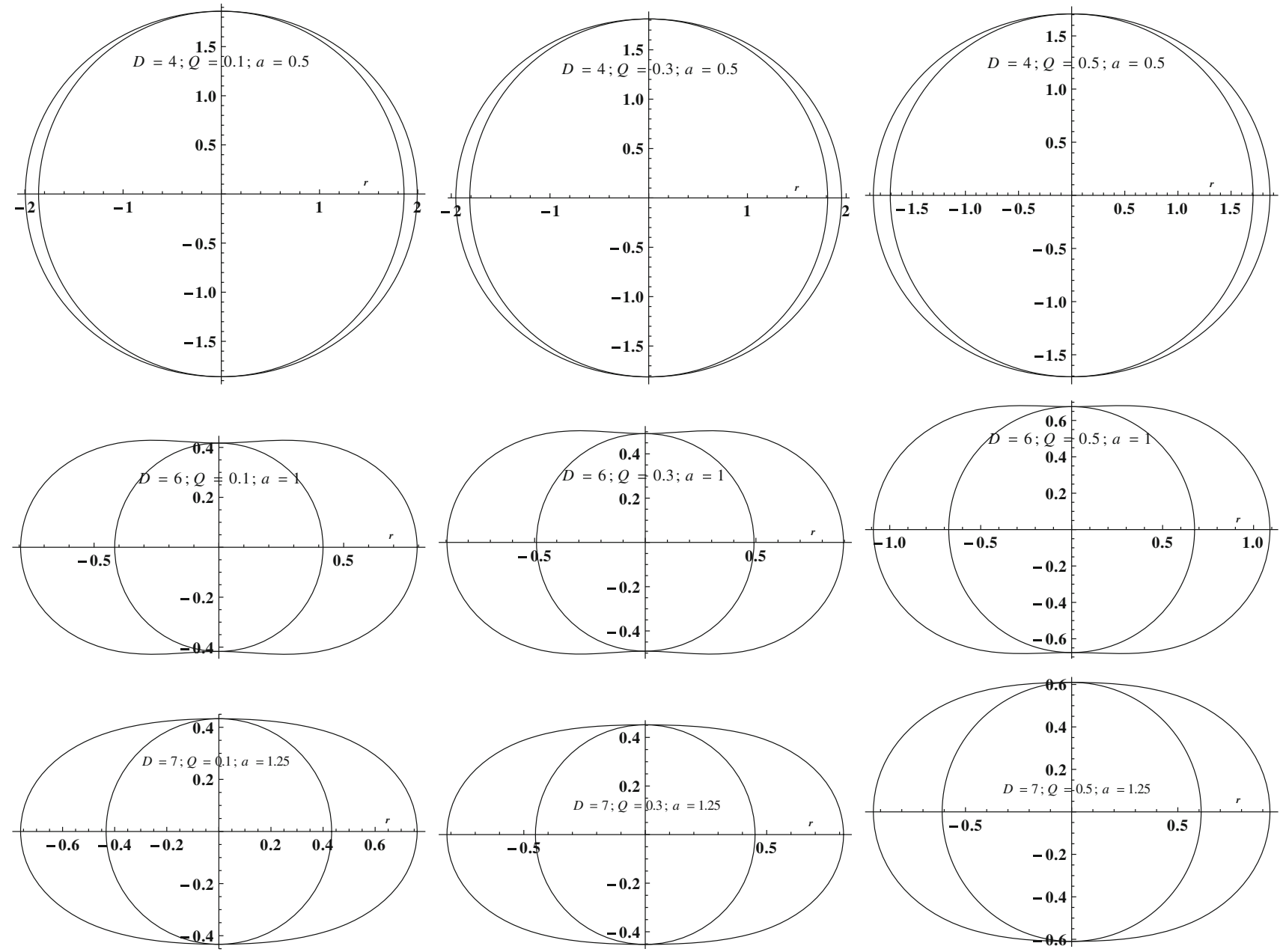

Fig. 4 Plots showing a cross section of SLS and EH and the variation of the ergosphere for different dimensions $D$ with three different values of the YM gauge charge parameter $Q$

(15) is stationary, axisymmetric, and asymptotically flat. It depends on the mass, the YM gauge charge, and the spinning parameter, which reduces to the $\operatorname{Kerr} \mathrm{BH}[1](N=1, Q=0)$ and the Myers-Perry BH [26] ( $Q=0)$. The solution in 4D $(N=1)$ has precisely the geometry of Kerr-Newman [5], but the charge that determines the geometry is the YM gauge charge. Also, it is easy to check that the metric (15) in 4D $(N=1)$ is a solution of the EYM (7). Thus, we can say that a spinning BH solution of the Einstein-Maxwell equations is also solution of EYM, but the charge $Q$ is the YM gauge charge and not the electric charge. However, this is not true in HD. Our spinning HD EYM BH solution deviates from the HD Kerr-Newman case [32] because $\Delta(r)$ for the latter is given by

$\Delta_{K N}(r)=r^{2}+a^{2}-\frac{\mu}{r^{N-2}}+\frac{Q^{2}}{r^{2(N-1)}}$.

The corresponding $\Delta(r)$ in a spinning HD EYM BH is given by (16). The difference in the last term of (16) and (34) is because of the fact that the charge term $Q^{2} / r^{2}$ in the solution (9) is dimension independent, while it would go as $Q^{2} / r^{2(N-1)}$ in HD Reissner-Nordström. It may be noted that HD Reissner-Nordström can be used to obtain HD KerrNewman using NJA [6]. The two kinds of horizon-like surfaces viz. SLS and EH are studied. In 4D, it turns out that there exist two horizon-like surfaces corresponding to two positive roots, which are identified as inner and outer horizons. However, in HD there exists only one positive root and thereby only one SLS and EH. It is interesting to see that the structure of the SLS, EH, and ergosphere are sensitive to the YM gauge charge parameter $Q$.

The physical properties of the solutions have not yet been fully investigated, this being a very severe job. However, we are currently working on this project. We have also shown that the presence of a YM gauge charge decreases the temperature with increase in gauge charge parameter Q. Such a change could have a significant effect in the thermodynamics of a BH. Hence, it will be of interest to see how the YM gauge 

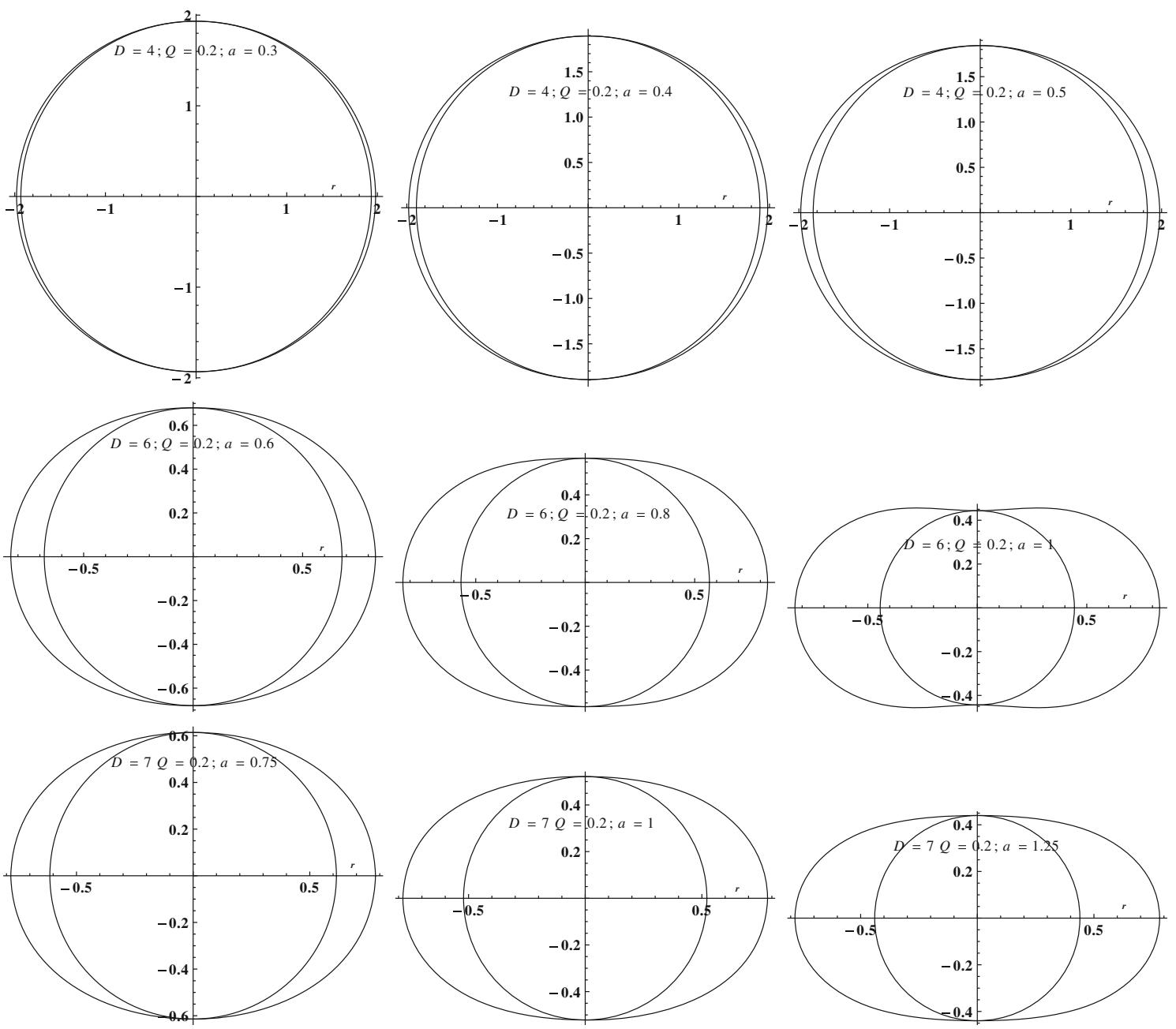

Fig. 5 Plots showing the cross sections of SLS and EH and the dependence of the shape of the ergosphere for different dimensions $D$ with three different values of the rotation parameter $a$

charge affects the thermodynamics by deriving a Smarr-like relation and the first law, and also analyzing stability. Further analysis of these solutions and the role of the YM gauge charge and spacetime dimension in an energy extraction process remains an interesting issue to explore in the future.

Acknowledgments The authors would like to thank the University Grant Commission (UGC) for major research project Grant No. F-39$459 / 2010$ (SR) and to IUCAA, Pune, for kind hospitality while part of this work was being done.

Open Access This article is distributed under the terms of the Creative Commons Attribution License which permits any use, distribution, and reproduction in any medium, provided the original author(s) and the source are credited.

Funded by $\mathrm{SCOAP}^{3}$ / License Version CC BY 4.0.

\section{References}

1. R.P. Kerr, Phys. Rev. Lett. D 11, 237 (1963)

2. F.R. Tangherlini, Nuovo Cim. 27, 636 (1963)
3. E.T. Newman, A.I. Janis, J. Math. Phys. 6, 915 (1965)

4. G.W. Gibbons, S.W. Hawking, Phys. Rev. D 15, 2738 (1977)

5. E.T. Newman, R. Couch, K. Chinnapared, A. Exton, A. Prakash, R. Torrence, J. Math. Phys. 6, 918 (1965)

6. D.Y. Xu, Class. Quant. Grav. 5, 871 (1988)

7. S.P. Drake, R. Turolla, Class. Quant. Grav. 14, 1883 (1997)

8. H. Kim, Phys. Rev. D 59, 064002 (1999)

9. S. Yazadjiev, Gen. Rel. Grav. 32, 2345 (2000)

10. S.P. Drake, P. Szekeres, Gen. Rel. Grav. 32, 445 (2000)

11. D.J. Cirilo Lombardo, Class. Quant. Grav. 21, 1407 (2004)

12. F. Caravelli, L. Modesto, Class. Quant. Grav. 27, 245022 (2010)

13. L. Modesto, P. Nicolini, Phys. Rev. D 82, 104035 (2010)

14. S. Capozziello, M. De laurentis, A. Stabile, Class. Quant. Grav. 27, 165008 (2010)

15. T. Johannsen, D. Psaltis, Phys. Rev. D 83, 124015 (2011)

16. C. Bambi, L. Modesto, Phys. Lett. B 721, 329 (2013)

17. E.N. Glass, J.P. Krisch, arXiv:0405143 [gr-qc]

18. R. d'Inverno, Introducing Einstein's Relativity (Clarendon, Oxford, 1992)

19. E.J. Flaherty, Hermitian and Kahlerian Geometry in Relativity Lecture notes in Physics, vol. 46 (Springer, Berlin, 1976)

20. M.M. Schiffer, R.J. Adler, J. Mark, C. Scheffield, J. Math. Phys. 14, 52 (1973) 
21. A. Strominger, C. Vafa, Phys. Lett. B 379, 99 (1996)

22. R. Emparan, S.R. Harvey, Living Rev. Relativ. 11, 6 (2008)

23. P. Kanti, Int. J. Mod. Phys. A 19, 4899 (2004)

24. A. Chados, S. Detweiler, Gen. Relativ. Gravit. 14, 879 (1982)

25. A. Das, A. DeBenedictis, Progr. Theo. Phys. 108, 119 (2002)

26. R.C. Myers, M.J. Perry, Ann. Phys. 172, 304 (1986)

27. T.T. Wu, C.N. Yang, in Properties of Matter Under Unusual Conditions, ed. by H. Mark, S. Fernbach (Interscience, New York, 1969), p. 349

28. P.B. Yasskin, Phys. Rev. D 12, 2212 (1975)

29. S. Habib Mazharimousavi, M. Halilsoy, Phys. Rev. D 76, 087501 (2007)
30. S. Habib Mazharimousavi, M. Halilsoy, JCAP 0812, 005 (2008)

31. S.H. Mazharimousavi, M. Halilsoy, Phys. Lett. B 659, 471 (2008)

32. A.N. Aliev, Phys. Rev. D 74, 024011 (2006)

33. A.N. Aliev, gr-qc/0612169 (2006)

34. S.G. Ghosh, N. Dadhich, Phys. Rev. D 82, 044038 (2010)

35. R. Banerjee, B.R. Majhi, S.K. Modak, S. Samanta, Phys. Rev. D 82, $124002(2010)$

36. J.D. Bekenstein, Phys. Rev. D 7, 2333 (1973)

37. R. Penrose, R.M. Floyd, Nature 229, 177 (1971) 